\title{
Comparative activity of newer $\beta$-lactam $/ \beta$-lactamase inhibitor combinations against Pseudomonas aeruginosa from patients hospitalized with pneumonia in European medical centers in 2020
}

\author{
Helio S. Sader ${ }^{1} \cdot$ Cecilia G. Carvalhaes $^{1} \cdot$ Dee Shortridge $^{1} \cdot$ Mariana Castanheira $^{1}$
}

Received: 27 August 2021 / Accepted: 5 October 2021 / Published online: 16 October 2021

(c) The Author(s) 2021

\begin{abstract}
Pseudomonas aeruginosa isolates were consecutively collected from patients with pneumonia in 29 medical centers in 2020 and susceptibility tested by broth microdilution method. Ceftazidime-avibactam (95.5\% susceptible), imipenem-relebactam (94.3\% susceptible), and ceftolozane-tazobactam (93.3\% susceptible) were the most active compounds after colistin (99.5\% susceptible). Susceptibility rates for the $\beta$-lactam/ $\beta$-lactamase inhibitor combinations (BL/BLIs) varied against isolates resistant to piperacillin-tazobactam, meropenem, imipenem, and/or ceftazidime. Ceftazidime-avibactam was the most active BL/BLI against resistant subsets from Western Europe, whereas imipenem-relebactam was slightly more active than other BL/BLIs against resistant subsets from Eastern Europe. Susceptibility rates were markedly lower in Eastern Europe than Western Europe.
\end{abstract}

Keywords Pseudomonas aeruginosa · Ceftazidime-avibactam · Ceftolozane-tazobactam · Imipenem-relebactam · Meropenem-vaborbactam

\section{Introduction}

The initial antimicrobial therapy of patients with pneumonia is frequently empirical, and the most appropriate empirical regimen is determined mainly by understanding causative pathogens and the antimicrobial susceptibility of these organisms. Moreover, the implementation of timely and effective antimicrobial therapy is critical to decrease complications and mortality [1-3].

Pseudomonas aeruginosa is one of the most common organisms isolated from respiratory samples of patients with pneumonia in European medical centers [3, 4] and represents a serious therapeutic challenge because it exhibits intrinsically decreased susceptibility to a range of antimicrobials and possesses a great ability to acquire and/or develop a diversity of resistant traits that can affect one or multiple antimicrobial agents $[5,6]$. P. aeruginosa carries an inducible AmpC cephalosporinase, which can cause resistance

Helio S. Sader

helio-sader@jmilabs.com

1 JMI Laboratories, 345 Beaver Kreek Centre, Suite A, North Liberty, IA 52317, USA to anti-pseudomonal cephalosporins and piperacillin-tazobactam when its production is significantly increased. Furthermore, upregulation of MexA-MexB-OprM and the loss of OprD are considered the most prevalent mechanisms of carbapenem resistance in $P$. aeruginosa; these mechanisms are usually associated with AmpC hyperproduction [7]. It is also important to note that infections caused by multidrugresistant (MDR) P. aeruginosa strains and a delay in appropriate antimicrobial therapy for serious $P$. aeruginosa infections are associated with longer hospital stays and increased mortality [8].

The most prominent group of new antimicrobial agents with broad spectrum activity is the $\beta$-lactam/ $\beta$-lactamase inhibitor combinations (BL/BLI). Four such combinations have been approved in recent years: ceftazidime-avibactam, ceftolozane-tazobactam, meropenem-vaborbactam, and imipenem-relebactam. Many others are currently in different stages of development and approval [9]. In this study, we evaluated the in vitro activity of these 4 most recently approved BL/BLIs against $P$. aeruginosa isolates recovered from respiratory samples of patients hospitalized with pneumonia in European hospitals in 2020. 


\section{Materials and methods}

Bacterial isolates were collected via the SENTRY Antimicrobial Surveillance Program and sent to JMI Laboratories (North Liberty, IA, USA) for susceptibility testing [10]. Each participating center was asked to collect 100 consecutive bacterial isolates from respiratory specimens determined to be significant by local criteria as the reported probable cause of pneumonia. Qualified sputum samples and isolates from invasive sampling, such as transtracheal aspiration, bronchoalveolar lavage, and protected brush samples, were accepted.

A total of 2,793 bacterial isolates were collected in 2020, including the $583 P$. aeruginosa evaluated in this study. Isolates were collected from 29 medical centers located in Western Europe (W-EU; $n=401 ; 21$ centers in 10 countries [Belgium, France, Germany, Ireland, Italy, Portugal, Spain, Sweden, Switzerland, and the UK]) and the Eastern European and Mediterranean region (E-EU; $n=182 ; 8$ centers in 8 countries [Czech Republic, Greece, Hungary, Israel, Poland, Romania, Slovenia, and Turkey]). Species identification was confirmed by using standard biochemical tests and/or a MALDI Biotyper (Bruker Daltonics, Billerica, MA, USA), when necessary.

All isolates were susceptibility tested using the reference broth microdilution method at a monitoring laboratory (JMI Laboratories, North Liberty, IA, USA) as described by the CLSI [11]. Ceftazidime-avibactam, ceftolozane-tazobactam, imipenem-relebactam, and piperacillin-tazobactam were tested with the $\beta$-lactamase inhibitor at fixed concentration of $4 \mathrm{mg} / \mathrm{L}$; meropenem-vaborbactam was tested with vaborbactam at fixed concentration of $8 \mathrm{mg} / \mathrm{L}[11,12]$. MIC results were interpreted according to EUCAST breakpoint criteria [13].

\section{Results}

Overall, ceftazidime-avibactam $\left(\mathrm{MIC}_{50 / 90}, 2 / 8 \mathrm{mg} / \mathrm{L}\right.$; $95.5 \%$ susceptible), imipenem-relebactam $\left(\mathrm{MIC}_{50 / 90}\right.$, $0.25 / 2 \mathrm{mg} / \mathrm{L} ; 94.3 \%$ susceptible), and ceftolozane-tazobactam $\left(\mathrm{MIC}_{50 / 90}, 0.5 / 2 \mathrm{mg} / \mathrm{L} ; 93.3 \%\right.$ susceptible) were the most active compounds against $P$. aeruginosa isolates after colistin (99.5\% susceptible; Table 1). All four newer BL/ BLIs were active against $>90 \%$ of $P$. aeruginosa isolates from W-EU. According to current EUCAST breakpoints criteria, ceftazidime-avibactam $\left(\mathrm{MIC}_{50 / 90}, 2 / 4 \mathrm{mg} / \mathrm{L}\right)$ showed the highest susceptibility rate (97.2\%) against isolates from $\mathrm{W}-\mathrm{EU}$, followed by imipenem-relebactam $\left(\mathrm{MIC}_{50 / 90}, 0.25 / 1 \mathrm{mg} / \mathrm{L} ; 94.5 \%\right.$ susceptible), ceftolozanetazobactam $\left(\mathrm{MIC}_{50 / 90}, 0.5 / 2 \mathrm{mg} / \mathrm{L} ; 94.3 \%\right.$ susceptible), and meropenem-vaborbactam $\left(\mathrm{MIC}_{50 / 90}, 0.5 / 8 \mathrm{mg} / \mathrm{L}\right.$; $91.0 \%$ susceptible; Table 1). It is important to note that the higher susceptibility rates of meropenem-vaborbactam in comparison with meropenem is a result of the different breakpoints applied to the 2 compounds $(\leq 2 \mathrm{mg} / \mathrm{L}$ for meropenem and $\leq 8 \mathrm{mg} / \mathrm{L}$ for meropenem/vaborbactam) rather than a substantial improvement in activity, as evidenced by the near-identical $\mathrm{MIC}_{50 / 90}$ and percentage of resistance values (Table 1).

In general, susceptibility rates were slightly lower among isolates from E-EU, and the highest susceptibility rate was shown by imipenem-relebactam $\left(\mathrm{MIC}_{50 / 90}, 0.25 / 2 \mathrm{mg} / \mathrm{L}\right.$; $94.0 \%$ susceptible), followed by ceftazidime-avibactam $\left(\mathrm{MIC}_{50 / 90}, 2 / 8 \mathrm{mg} / \mathrm{L} ; 91.8 \%\right.$ susceptible), ceftolozanetazobactam $\left(\mathrm{MIC}_{50 / 90}, 0.5 / 4 \mathrm{mg} / \mathrm{L} ; 91.2 \%\right.$ susceptible), and meropenem-vaborbactam $\left(\mathrm{MIC}_{50 / 90}, 0.5 / 16 \mathrm{mg} / \mathrm{L} ; 83.5 \%\right.$ susceptible; Table 1). The most active comparator agents were colistin $(99.7 \%$ and $98.9 \%$ susceptible in W-EU and E-EU, respectively), amikacin (94.0\% and $87.9 \%$ susceptible in W-EU and E-EU, respectively), and tobramycin (91.8\% and $84.6 \%$ susceptible in $\mathrm{W}-\mathrm{EU}$ and E-EU, respectively; Table 1).

Susceptibility rates for the BL/BLIs varied more broadly against isolates resistant to piperacillin-tazobactam, meropenem, imipenem, or ceftazidime (Table 2). Ceftazidimeavibactam was the most active BL/BLI against these resistant subsets from $\mathrm{W}$-EU, with susceptibility rates ranging from $92.6 \%$ when tested against imipenem-resistant isolates to $87.8 \%$ against ceftazidime-resistant strains. Imipenemrelebactam was slightly more active than the other BL/BLIs against resistant subsets from E-EU. Imipenem-relebactam susceptibility rates against resistant subsets from E-EU ranged from $81.4 \%$ against imipenem-resistant isolates to $64.5 \%$ against meropenem-resistant strains (Table 2). Moreover, ceftazidime-avibactam retained activity against $81.2 \%$ of W-EU isolates resistant to piperacillin-tazobactam, imipenem, meropenem, and ceftazidime, whereas ceftolozanetazobactam, imipenem-relebactam, and meropenem-vaborbactam were active against $59.0 \%, 53.8 \%$, and $7.7 \%$ of these isolates, respectively (Table 2). Imipenem-relebactam was the most active agent against E-EU isolates resistant to these $4 \beta$-lactam compounds, inhibiting $64.5 \%$ at the EUCAST susceptible breakpoint of $\leq 2 \mathrm{mg} / \mathrm{L}$ (Table 2).

\section{Discussion}

The treatment of $P$. aeruginosa pneumonia represents a great challenge for physicians. $P$. aeruginosa is an organism for which very few therapeutic options are clinically available, and resistance to tractional anti-pseudomonal $\beta$-lactams, such as piperacillin-tazobactam, ceftazidime, cefepime, imipenem, and meropenem, is elevated in many geographic 


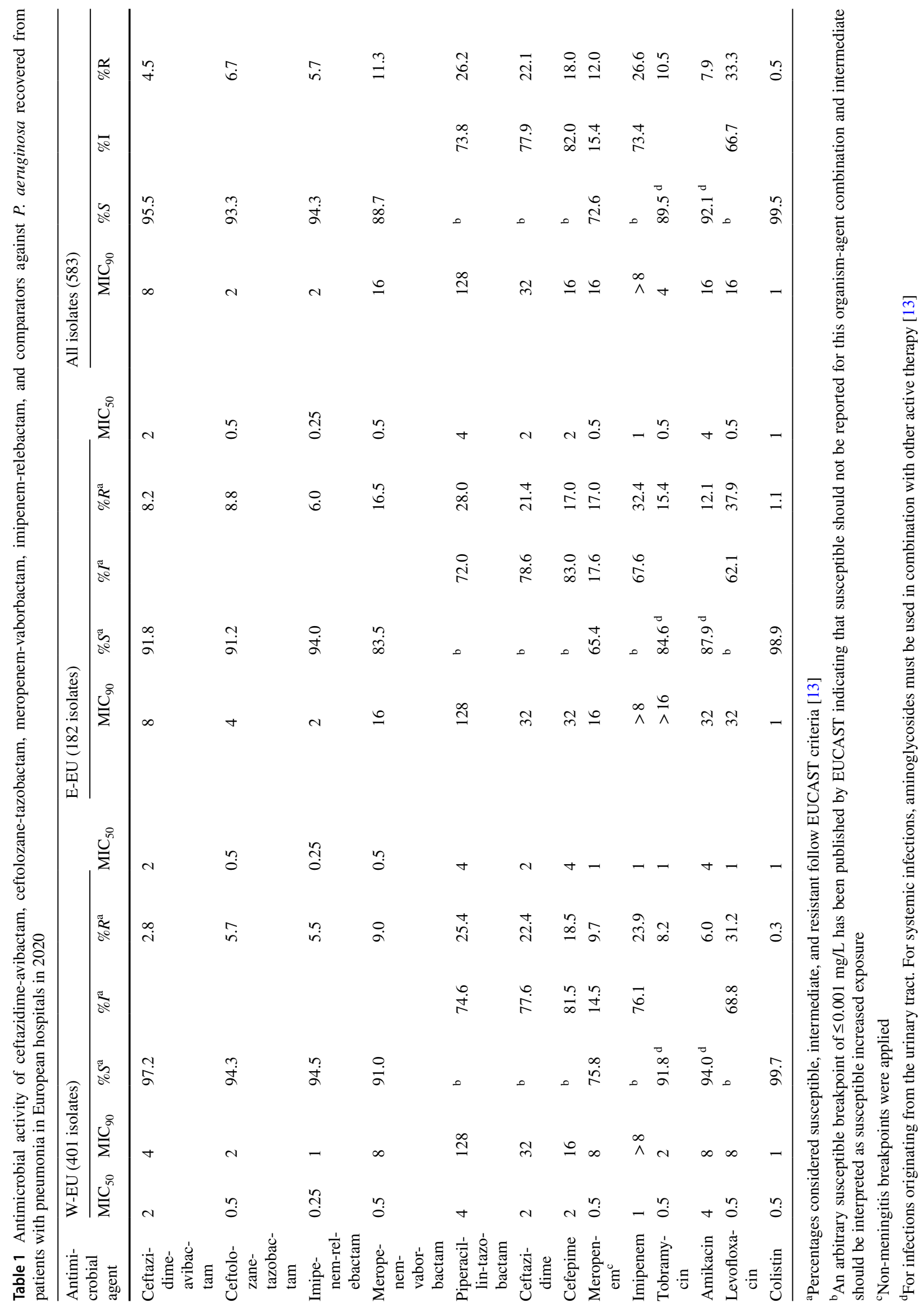


Table 2 Antimicrobial activity of ceftazidime-avibactam, ceftolozane-tazobactam, meropenem-vaborbactam, imipenem-relebactam, and comparators against resistant subsets of $P$. aeruginosa isolates from patients with pneumonia in European hospitals in 2020

\begin{tabular}{|c|c|c|c|}
\hline \multirow[t]{2}{*}{ Resistant phenotype } & \multicolumn{3}{|c|}{$\begin{array}{l}\text { \% Susceptible per EUCAST } \\
\text { (no. of isolates) }\end{array}$} \\
\hline & W-EU & E-EU & All isolates \\
\hline $\begin{array}{l}\text { Piperacillin-tazobactam-resistant } \\
\text { (MIC > } 16 \mathrm{mg} / \mathrm{L})\end{array}$ & $(102)$ & $(51)$ & $(153)$ \\
\hline Ceftazidime-avibactam & 91.2 & 70.6 & 84.3 \\
\hline Ceftolozane-tazobactam & 78.4 & 70.0 & 75.7 \\
\hline Imipenem-relebactam & 83.3 & 78.4 & 81.7 \\
\hline Meropenem-vaborbactam & 69.6 & 49.0 & 62.7 \\
\hline Meropenem-resistant (MIC > $8 \mathrm{mg} / \mathrm{L}$ ) & (39) & (31) & (70) \\
\hline Ceftazidime-avibactam & 81.6 & 54.8 & 69.9 \\
\hline Ceftolozane-tazobactam & 59.0 & 54.8 & 57.1 \\
\hline Imipenem-relebactam & 53.8 & 64.5 & 58.6 \\
\hline Meropenem-vaborbactam & 7.7 & 3.2 & 5.7 \\
\hline Imipenem-resistant (MIC > 4 mg/L) & $(96)$ & (59) & $(155)$ \\
\hline Ceftazidime-avibactam & 92.6 & 78.0 & 87.0 \\
\hline Ceftolozane-tazobactam & 84.4 & 78.0 & 81.9 \\
\hline Imipenem-relebactam & 77.1 & 81.4 & 78.7 \\
\hline Meropenem-vaborbactam & 63.5 & 52.5 & 59.4 \\
\hline Ceftazidime-resistant (MIC > 8 mg/L) & $(90)$ & $(39)$ & $(129)$ \\
\hline Ceftazidime-avibactam & 87.8 & 61.5 & 79.8 \\
\hline Ceftolozane-tazobactam & 75.6 & 61.5 & 71.3 \\
\hline Imipenem-relebactam & 81.1 & 74.4 & 79.1 \\
\hline Meropenem-vaborbactam & 72.2 & 46.2 & 64.3 \\
\hline$\beta$-lactam-resistant ${ }^{\mathrm{a}}$ & $(16)$ & (9) & $(25)$ \\
\hline Ceftazidime-avibactam & 81.2 & 33.3 & 64.0 \\
\hline Ceftolozane-tazobactam & 43.8 & 55.6 & 48.0 \\
\hline Imipenem-relebactam & 43.8 & 55.6 & 48.0 \\
\hline Meropenem-vaborbactam & 6.2 & 0.0 & 4.0 \\
\hline
\end{tabular}

${ }^{a}$ Isolates resistant to piperacillin-tazobactam $(\mathrm{MIC}>16 \mathrm{mg} / \mathrm{L})$, meropenem $(\mathrm{MIC}>8 \mathrm{mg} / \mathrm{L}$ ), imipenem(MIC $>4 \mathrm{mg} / \mathrm{L})$, and ceftazidime (MIC $>8 \mathrm{mg} / \mathrm{L}$ ) per EUCAST criteria [13]

Abbreviations: $W$-EU Western Europe, $E-E U$ Eastern Europe

regions. Moreover, non- $\beta$-lactam agents that are active against $P$. aeruginosa, such as aminoglycosides, colistin, and fosfomycin, are limited in their efficacy, safety profile, and/or by the emergence of resistance $[2,7,8]$.

Novel BL/BLIs represent valuable new therapeutic options for $P$. aeruginosa infections, for which limited treatment options were available [9]. In the present study, we evaluated the antimicrobial susceptibility of contemporary (2020) isolates of $P$. aeruginosa recovered from respiratory samples of patients with pneumonia. Our results complement the results of other surveillance programs by providing comparative results for the four BL/BLIs most recently approved for the treatment of $P$. aeruginosa pneumonia in Europe [14-17]. Other surveillance networks, like the
European Antimicrobial Resistance Surveillance Network (EARS-NET), evaluates the antimicrobial susceptibility of $P$. aeruginosa in many European countries and publishes valuable data periodically, but the activities of these new BL/BLIs are not evaluated in EARS-NET or other large surveillance programs $[18,19]$.

The results of this investigation showed that, besides colistin, the new BL/BLIs were the most active compounds against $P$. aeruginosa, with susceptibility rates similar to the aminoglycosides tobramycin and amikacin. Our results also showed that these new BL/BLIs, especially ceftazidime-avibactam, imipenem-relebactam, and ceftolozane-tazobactam, retained good activity against $P$. aeruginosa isolates resistant to $\beta$-lactams currently used to treat $P$. aeruginosa infections.

Another interesting finding was the regional variation of the activity of these BL/BLIs within Europe. Ceftazidimeavibactam was the most active agent against isolates from W-EU with $97.2 \%$ susceptibility, followed by imipenemrelebactam (94.5\% susceptible) and ceftolozane-tazobactam (94.3\% susceptible). Imipenem-relebactam was the most active BL/BLI against isolates from E-EU (94.0\% susceptible), followed by ceftazidime-avibactam ( $91.8 \%$ susceptible) and ceftolozane-tazobactam (91.2\% susceptible; Table 1). Regional differences on the activities of these BL/BLIs reflect the variety of resistance mechanisms expressed by $P$. aeruginosa and illustrate how these mechanisms may have different impacts on each of these compounds. Mechanisms of resistance to these new BL/BLIs are usually very complex and caused by the presence and interaction of multiple mutation-driven resistance mechanisms [20, 21]. Therefore, the activity of these compounds, and especially the rates of cross-resistance between them, may vary widely depending on selective pressure due to previous antibiotic usage.

The limitations of the study should be considered when interpreting the results and conclusions. First, the criteria used to categorize a bacterial isolate as clinically significant were not defined in the study protocol and were based on local algorithms. Second, due to the lack of clinical information available, this study could not exclude the possibility that some organisms were colonizers. Third, a limited number of isolates and/or medical centers were surveyed in some European countries; thus, the results presented here may not represent the overall picture from those European regions.

In conclusion, the recently approved BL/BLIs demonstrated potent activity and broad coverage against $P$. aeruginosa isolated from patients with pneumonia in European medical centers. Based on the current EUCAST breakpoints, ceftazidime-avibactam, ceftolozane-tazobactam, and imipenem-relebactam showed similar overall coverage (\% susceptible) against $P$. aeruginosa, while susceptibility rates were lower for meropenem-vaborbactam, especially against resistant subsets. Moreover, susceptibility rates were markedly lower in E-EU compared to W-EU. 
Acknowledgements The authors thank all participants of the SENTRY Antimicrobial Surveillance Program for their work in providing isolates. Editorial support was provided by Amy Chen, Gauri Deshpande, and Judy Oberholser at JMI Laboratories and was funded by Pfizer.

Funding This study was supported by Pfizer Inc. Helio S. Sader, Cecilia G. Carvalhaes, Dee Shortridge, and Mariana Castanheira are employees of JMI Laboratories, which was paid consultant to Pfizer in connection with the development of this manuscript.

\section{Declarations}

Ethics approval Not required.

Conflict of interest The authors declare no competing interests.

Author disclosure statement JMI Laboratories contracted to perform services in 2018-2021 for Achaogen, Inc., Affinity Biosensors, Albany College of Pharmacy and Health Sciences, Allecra Therapeutics, Allergan, Amicrobe Advanced Biomaterials, Inc., American Proficiency Institute, AmpliPhi Biosciences Corp., Amplyx Pharma, Antabio, Arietis Corp., Arixa Pharmaceuticals, Inc., Artugen Therapeutics USA, Inc., Astellas Pharma Inc., Athelas, Becton, Basilea Pharmaceutica Ltd., Bayer AG, Becton, Beth Israel Deaconess Medical Center, BIDMC, bioMerieux, Inc., bioMerieux SA, BioVersys Ag, Boston Pharmaceuticals, Bugworks Research Inc., CEM-102 Pharmaceuticals, Cepheid, Cidara Therapeutics, Inc., Cipla, Contrafect, Cormedix Inc., Crestone, Inc., Curza, CXC7, DePuy Synthes, Destiny Pharma, Dickinson and Company, Discuva Ltd., Dr. Falk Pharma GmbH, Emery Pharma, Entasis Therapeutics, Eurofarma Laboratorios SA, Fedora Pharmaceutical, F. Hoffmann-La Roche Ltd., Fimbrion Therapeutics, US Food and Drug Administration, Fox Chase Chemical Diversity Center, Inc., Gateway Pharmaceutical LLC, GenePOC Inc., Geom Therapeutics, Inc., GlaxoSmithKline plc, Guardian Therapeutics, Hardy Diagnostics, Harvard University, Helperby, HiMedia Laboratories, ICON plc, Idorsia Pharmaceuticals Ltd., IHMA, Iterum Therapeutics plc, Janssen Research \& Development, Johnson \& Johnson, Kaleido Biosciences, KBP Biosciences, Laboratory Specialists, Inc., Luminex, Matrivax, Mayo Clinic, Medpace, Meiji Seika Pharma Co., Ltd., Melinta Therapeutics, Inc., Menarini, Merck \& Co., Inc., Meridian Bioscience Inc., Micromyx, Microchem Laboratory, MicuRx Pharmaceutics, Inc., Mutabilis Co., N8 Medical, Nabriva Therapeutics plc, National Institutes of Health, NAEJA-RGM, National University of Singapore, North Bristol NHS Trust, Novartis AG, Novome Biotechnologies, Oxoid Ltd., Paratek Pharmaceuticals, Inc., Pfizer, Inc., Pharmaceutical Product Development, LLC, Polyphor Ltd., Prokaryotics Inc., QPEX Biopharma, Inc., Ra Pharmaceuticals, Inc., Rhode Island Hospital, RIHML, Roche, Roivant Sciences, Ltd., Safeguard Biosystems, Salvat, Scynexis, Inc., SeLux Diagnostics, Inc., Shionogi and Co., Ltd., SinSa Labs, Specific Diagnostics, Spero Therapeutics, Summit Pharmaceuticals International Corp., SuperTrans Medical LT, Synlogic, T2 Biosystems, Taisho Pharmaceutical Co., Ltd., TenNor Therapeutics Ltd., Tetraphase Pharmaceuticals, The Medicines Company, The University of Queensland, Theravance Biopharma, Thermo Fisher Scientific, Tufts Medical Center, Universite de Sherbrooke, University of Colorado, University of Southern California-San Diego, University of Iowa, University of Iowa Hospitals and Clinics, University of North Texas Health Science Center, University of Wisconsin, UNT System College of Pharmacy, URMC, UT Southwestern, VenatoRx, Viosera Therapeutics, Vyome Therapeutics Inc., Wayne State University, Wockhardt, Yukon Pharmaceuticals, Inc., Zai Lab, and Zavante Therapeutics, Inc. There are no speakers' bureaus or stock options to declare.
Open Access This article is licensed under a Creative Commons Attribution 4.0 International License, which permits use, sharing, adaptation, distribution and reproduction in any medium or format, as long as you give appropriate credit to the original author(s) and the source, provide a link to the Creative Commons licence, and indicate if changes were made. The images or other third party material in this article are included in the article's Creative Commons licence, unless indicated otherwise in a credit line to the material. If material is not included in the article's Creative Commons licence and your intended use is not permitted by statutory regulation or exceeds the permitted use, you will need to obtain permission directly from the copyright holder. To view a copy of this licence, visit http://creativecommons.org/licenses/by/4.0/.

\section{References}

1. Nair GB, Niederman MS (2013) Nosocomial pneumonia: lessons learned. Crit Care Clin 29(3):521-546

2. Kalil AC, Metersky ML, Klompas M, Muscedere J, Sweeney DA, Palmer LB, Napolitano LM, O'Grady NP, Bartlett JG, Carratala J, El Solh AA, Ewig S, Fey PD, File TM Jr, Restrepo MI, Roberts JA, Waterer GW, Cruse P, Knight SL, Brozek JL (2016) Management of adults with hospital-acquired and ventilator-associated pneumonia: 2016 clinical practice guidelines by the Infectious Diseases Society of America and the American Thoracic Society. Clin Infect Dis 63(5):e61-e111

3. Koulenti D, Tsigou E, Rello J (2017) Nosocomial pneumonia in 27 ICUs in Europe: perspectives from the EU-VAP/CAP study. Eur J Clin Microbiol Infect Dis 36(11):1999-2006

4. Sader HS, Streit JM, Carvalhaes CG, Huband MD, Shortridge D, Mendes RE, Castanheira M (2021) Frequency of occurrence and antimicrobial susceptibility of bacteria isolated from patients hospitalized with bacterial pneumonia in western Europe, eastern Europe, and the United States: Results from the SENTRY Program (2016-2019). JAC Antimicrob Resist. https://doi.org/10. 1093/jacamr/dlab117

5. Langendonk RF, Neill DR, Fothergill JL (2021) The building blocks of antimicrobial resistance in Pseudomonas aeruginosa: implications for current resistance-breaking therapies. Front Cell Infect Microbiol 11:665759

6. Tamma PD, Aitken SL, Bonomo RA, Mathers AJ, van Duin D, Clancy CJ (2021) Infectious Diseases Society of America guidance on the treatment of extended-spectrum beta-lactamase producing Enterobacterales (ESBL-E), carbapenem-resistant Enterobacterales (CRE), and Pseudomonas aeruginosa with difficult-to-treat resistance (DTR-P. aeruginosa). Clin Infect Dis 72(7):1109-1116

7. Horcajada JP, Montero M, Oliver A, Sorlí L, Luque S, GómezZorrilla S, Benito N, Grau S (2019) Epidemiology and treatment of multidrug-resistant and extensively drug-resistant Pseudomonas aeruginosa infections. Clin Microbiol Rev 32(4):e00031-e119

8. Micek ST, Wunderink RG, Kollef MH, Chen C, Rello J, Chastre J, Antonelli M, Welte T, Clair B, Ostermann H, Calbo E, Torres A, Menichetti F, Schramm GE, Menon V (2015) An international multicenter retrospective study of Pseudomonas aeruginosa nosocomial pneumonia: impact of multidrug resistance. Crit Care 19:219

9. Yahav D, Giske CG, Gramatniece A, Abodakpi H, Tam VH, Leibovici L (2020) New beta-lactam-beta-lactamase inhibitor combinations. Clin Microbiol Rev 34(1):e00115

10. Sader HS, Castanheira M, Arends SJR, Goossens H, Flamm RK (2019) Geographical and temporal variation in the frequency and antimicrobial susceptibility of bacteria isolated from patients hospitalized with bacterial pneumonia: results from 20 years of 
the SENTRY Antimicrobial Surveillance Program (1997-2016). J Antimicrob Chemother 74(6):1595-1606

11. CLSI (2018) Methods for dilution antimicrobial susceptibility tests for bacteria that grow aerobically: eleventh edition M07. Clinical and Laboratory Standards Institute, Wayne, PA

12. CLSI (2021) M100Ed31. Performance standards for antimicrobial susceptibility testing: 31 st informational supplement. Clinical and Laboratory Standards Institute, Wayne, PA

13. EUCAST (2021) Breakpoint tables for interpretation of MICs and zone diameters. Version 11.0, January 2021. European Committee on Antimicrobial Susceptibility Testing. Availabel at https://www. eucast.org/fileadmin/src/media/PDFs/EUCAST_files/Breakpoint_ tables/v_11.0_Breakpoint_Tables.pdf. Accessed Jul 2021

14. (2021) Recarbrio® (imipemen/cilastatin/relebactam). https:// www.ema.europa.eu/en/documents/product-information/recar brio-epar-product-information_en.pdf. Accessed Jul 2021

15. (2021) Vaborem ${ }^{\circledR}$ (meropenem-vaborbactam). https://www.ema. europa.eu/en/documents/product-information/vaborem-eparproduct-information_en.pdf. Accessed Jul 2021

16. (2021) Zavicefta ${ }^{\circledR}$ (ceftazidime-avibactam). https://www.ema. europa.eu/en/documents/product-information/zavicefta-eparproduct-information_en.pdf. Accessed Jul 2021

17. (2021) Zerbaxa $®($ ceftolozane-tazobactam). Available at: https:// www.ema.europa.eu/en/documents/product-information/zerbaxaepar-product-information_en.pdf. Accessed Jul 2021

18. Antimicrobial resistance in the EU/EEA (EARS-Net) - Annual Epidemiological Report for 2019. European Centre for Disease
Prevention and Control. November 18 2020. https://www.ecdc. europa.eu/en/publications-data/surveillance-antimicrobial-resis tance-europe-2019\#no-link

19. Weiner-Lastinger LM, Abner S, Edwards JR, Kallen AJ, Karlsson M, Magill SS, Pollock D, See I, Soe MM, Walters MS, Dudeck MA (2020) Antimicrobial-resistant pathogens associated with adult healthcare-associated infections: summary of data reported to the National Healthcare Safety Network, 2015-2017. Infect Control Hosp Epidemiol 41(1):1-18

20. Castanheira M, Doyle TB, Smith CJ, Mendes RE, Sader HS (2019) Combination of MexAB-OprM overexpression and mutations in efflux regulators, PBPs and chaperone proteins is responsible for ceftazidime/avibactam resistance in Pseudomonas aeruginosa clinical isolates from US hospitals. J Antimicrob Chemother 74(9):2588-2595

21. Fraile-Ribot PA, Cabot G, Mulet X, Perianez L, Martin-Pena ML, Juan C, Perez JL, Oliver A (2018) Mechanisms leading to in vivo ceftolozane/tazobactam resistance development during the treatment of infections caused by MDR Pseudomonas aeruginosa. J Antimicrob Chemother 73(3):658-663

Publisher's note Springer Nature remains neutral with regard to jurisdictional claims in published maps and institutional affiliations. 